\title{
Upper bound on the secret key rate distillable from effective quantum correlations with imperfect detectors
}

\author{
Tobias Moroder, Marcos Curty, and Norbert Lütkenhaus \\ Quantum Information Theory Group, Institut für Theoretische Physik I, \\ and Max-Planck Research Group, Institute of Optics, Information and Photonics, \\ Universität Erlangen-Nürnberg, Staudtstraße 7/B2, 91058 Erlangen, Germany
}

(Dated: February 26, 2019)

\begin{abstract}
We provide a simple method to obtain an upper bound on the secret key rate that is particularly suited to analyze practical realizations of quantum key distribution protocols with imperfect devices. We consider the so-called trusted device scenario where Eve cannot modify the actual detection devices employed by Alice and Bob. The upper bound obtained is based on the available measurements results, but it includes the effect of the noise and losses present in the detectors of the legitimate users.

PACS numbers: 03.67.Dd, 03.65.Ud, 03.67.Mn
\end{abstract}

\section{INTRODUCTION}

Quantum key distribution (QKD) [1, 2] is a technique that allows two parties, typically called Alice and Bob, to generate an unconditionally secure secret key. This secret key can then be used together with the Vernam cipher [3] to achieve unconditionally secure communications against any possible eavesdropper, named Eve.

Typical practical QKD protocols distinguish two phases in order to generate a secret key. In the first phase, an effective bipartite quantum state is distributed between Alice and Bob. This state creates correlations between them and it might contain as well hidden correlations with Eve. Next, Alice and Bob perform a set of measurements on the given signal states in order to measure these correlations. As a result, they obtain a joint probability distribution $p\left(a_{i}, b_{j}\right) \equiv p_{i j}$ describing their classical outcomes. In the second phase, Alice and Bob try to distill a secret key from these correlated data $p_{i j}$ by means of public discussions over an authenticated classical channel. This procedure includes postselection of data, error correction to reconcile the data, and privacy amplification to decouple the data from Eve [4]. In this second phase, no quantum-mechanical manipulations are performed; it is a completely classical process.

Two types of schemes are used to create the correlated data in the first phase of QKD. In entanglement-based (EB) schemes, a source, which is assumed to be under Eve's control, produces a bipartite quantum state $\rho_{A B}$ that is distributed to Alice and Bob. Eve could even have a third system entangled with those given to the legitimate users. Alice and Bob measure each incoming signal by means of two positive operator valued measures (POVM) $\left\{A_{i}\right\}$ and $\left\{B_{j}\right\}$, respectively. While the subsystems measured by Alice and Bob result in correlations described by a joint probability distribution $p_{i j}$, Eve can use her subsystem to obtain information about the data of the legitimate users.

In prepare and measure (PM) schemes, Alice prepares a state $\left|\varphi_{i}\right\rangle$ with probability $p_{i}$ and sends it to Bob. On the receiving side, Bob measures each received signal with a
POVM described by the quantum operators $\left\{B_{j}\right\}$. Generalizing the ideas introduced by Bennett et al. [5], the signal preparation process in PM schemes can be thought of as follows: Alice produces first the bipartite quantum state $\left|\psi_{\text {source }}\right\rangle_{A B}=\sum_{i} \sqrt{p_{i}}\left|\alpha_{i}\right\rangle_{A}\left|\varphi_{i}\right\rangle_{B}$ and, afterward, she measures the first subsystem in the orthogonal basis $\left|\alpha_{i}\right\rangle_{A}$. This action generates the (nonorthogonal) signal states $\left|\varphi_{i}\right\rangle$ with probabilities $p_{i}$. It is important to note that in PM schemes, the reduced density matrix of Alice, $\rho_{A}=\operatorname{Tr}_{B}\left(\left|\psi_{\text {source }}\right\rangle_{A B}\left\langle\psi_{\text {source }}\right|\right)$, is fixed and cannot be modified by Eve. The state $\rho_{A}$ depends only on the probabilities $p_{i}$ and on the overlap of the signals states $\left|\varphi_{i}\right\rangle$. This information is known to all the parties. To include it in the measurement process one can add to the observables $\left\{A_{i} \otimes B_{j}\right\}$ measured by Alice and Bob other observables $\left\{C_{k} \otimes \mathbb{1}\right\}$ such that the observables $\left\{C_{k}\right\}$ form a tomographically complete set of Alice's Hilbert space [6].

From now on, we will always use the term entanglement and detection devices without making any further distinction between these two different QKD schemes. Moreover, $p_{i j}$ and $\left\{A_{i} \otimes B_{j}\right\}$ refer to the complete set of measurements, i.e., they include also the observables $\left\{C_{k} \otimes \mathbb{1}\right\}$ for PM schemes.

An essential question in QKD is whether the correlated data contained in $p_{i j}$ allow Alice and Bob to generate a secret key. In Ref. [7], it has been proven that a necessary precondition for secure key distribution is the provable presence of quantum correlations in $p_{i j}$. In this context, see also Ref. 8]. Given the set of measurements performed by Alice and Bob together with the resulting probabilities $p_{i j}$, the legitimate users can calculate the equivalence class $\mathcal{S}$ of quantum states that are compatible with the observed data $p_{i j}$ :

$$
\mathcal{S}=\left\{\rho_{A B} \mid \operatorname{Tr}\left(A_{i} \otimes B_{j} \rho_{A B}\right)=p_{i j}, \forall i, j\right\} .
$$

By definition, every state $\rho_{A B} \in \mathcal{S}$ can represent the state shared by Alice and Bob before their measurements [9]. According to Ref. 7], in order to be able to distill a secret key from the observed data, it is necessary to prove 
that the equivalence class $\mathcal{S}$ does not contain any separable state. See also Ref. [8]. This task is called effective entanglement verification, where the word "effective" is used to emphasize that the entanglement which might be contained in the state $\rho_{A B}$ is destroyed by Alice and Bob after their measurements or might not have been present at all in PM schemes. To deliver this entanglement proof, one can employ any separability criteria [10]. One possibility particularly suited for this purpose is the use of entanglement witnesses 6, 7].

From Ref. 7], we learn that, once the first phase of QKD is complete, we need to find quantum-mechanical correlations in $p_{i j}$. As it is, the effective entanglement verification answers only partially the important question of how much secret key can Alice and Bob obtain from their correlated data: it just tells if the secret key rate $K_{S}$ is zero or it may be positive. The problem of finding upper bounds for $K_{S}$ was recently analyzed from an entanglement theory point of view by K. Horodecki et al. in Ref. 11]. These authors showed that the value of $K_{S}$ that can be extracted from a quantum state $\rho_{A B}$ can be strictly greater than the distillable entanglement [13], and it is generally bounded from above by the regularized relative entropy of entanglement $E_{r}^{\infty}\left(\rho_{A B}\right)$ [1], 12]. This upper bound takes only into account the form of $\rho_{A B}$ and assumes perfect detection devices for Alice and Bob. To calculate the regularized relative entropy of entanglement of a given quantum state is, in general, a quite difficult task, and analytical expressions are only available for some particular states [14]. Another wellknown upper bound on $K_{S}$ is the so-called intrinsic information proposed by Maurer and Wolf [20]. See also Refs. 7, 15, 16]. In this case, one can easily incorporate the possible imperfections of the detectors in the upper bound. However, it requires to solve an infimum ranging over the set of all possible discrete probability distributions, or discrete channels, which is in principle not easy to compute. (See also Ref. [17].)

In this paper, we present a simple method to obtain an upper bound on $K_{S}$ that is particularly suited to evaluate the secret key rate on practical realizations of QKD schemes. Moreover, this procedure has the advantage that is is straightforward to calculate. It is based on the correlated data $p_{i j}$, and it also monitors any form of detector imperfections. Specifically, we consider the so-called trusted device scenario where Eve cannot modify the actual detection devices employed by Alice and Bob. We assume that the legitimate users have complete knowledge about their detectors, which are fixed by the actual experiment. These detectors might be noisy and might introduce losses and they are characterized by the POVMs $\left\{A_{i}\right\}$ and $\left\{B_{j}\right\}$.

The paper is organized as follows. In Sec. II, we introduce two desired properties for any upper bound on $K_{S}$ for practical QKD: effective entanglement verification, and the ability to monitor any kind of imperfections in Alice's and Bob's detection setups. This section contains as well a brief summary of some known upper bounds on $K_{S}$. Section III includes the main result of the paper: There we introduce a straightforward method to obtain an upper bound on $K_{S}$ that satisfies the conditions presented in Sec. II. This result is then illustrated in Sec. IV for two well-known QKD protocols with imperfect detectors: the four-state [2] and the six-state [18] QKD schemes. Finally, Sec. V concludes the paper with a summary.

\section{DESIRED PROPERTIES AND KNOWN UPPER BOUNDS ON $K_{S}$}

\section{A. Desired properties}

The secret key rate $K_{S}$, i.e., the rate of secret key that can be obtained per signal state sent by Alice to Bob, is one of the most important figures of merit in order to compare the performance of different QKD schemes. Ideally, one would like to calculate the maximum achievable value of $K_{S}$ from the data available once the first phase of the QKD protocol is completed. Note that the second phase is just a classical procedure that is completely independent of the actual experimental setup used.

In a similar spirit, any upper bound on the achievable secret key rate $K_{S}$ should as well be valid for any arbitrary public communication protocol performed during the second phase of QKD. The upper bound only depends on the observed data $p_{i j}$ together with the particular POVMs $\left\{A_{i}\right\}$ and $\left\{B_{j}\right\}$ used by Alice and Bob.

This gives rise to one main requirement expected from any upper bound on $K_{S}$ : if the observed data $p_{i j}$ can originate from a separable state, then any upper bound must vanish [7]. That is, the upper bound might be considered as the generalization of the necessary effective entanglement verification.

From a practical point of view, it would be necessary that any upper bound on $K_{S}$ includes the effect of imperfect devices. Especially, it should be able to take into account the low detection efficiency and the noise in the form of dark counts introduced by current detection devices. This fact is of special importance in order to compare different realizations of QKD, and it can be used to see the tradeoff between desired and realizable implementations of QKD.

\section{B. Known upper bounds on $K_{S}$}

In this section, we review very briefly some known upper bounds on $K_{S}$ that apply to particular scenarios:

Mutual information $I(A ; B)$ : Suppose Alice and Bob are connected by a public channel and have access to repeated independent realizations of two random variables, denoted as $A$ and $B$, and which are characterized by a joint probability distribution $p_{A B}$. An upper bound on the secret key rate $K_{S}$ is given by the mutual information $I(A ; B)$ [19, 20]. This quantity 
is defined in terms of the Shannon entropy $H(X)=$ $-\sum_{x \in X} p(x) \log p(x)$ and the Shannon joint entropy $H(X, Y)=-\sum_{x \in X} \sum_{y \in Y} p(x, y) \log p(x, y)$ as

$$
I(A ; B)=H(A)+H(B)-H(A, B) .
$$

This result is not surprising since the mutual information quantifies the reduction in the uncertainty of the random variable $A$ because of the knowledge of $B$.

This upper bound can directly be used for the case of QKD, just by taking as $p_{A B}$ the correlated data $p_{i j}$. Moreover, in this case, one can easily incorporate the effect of the imperfections in the detectors when calculating $I(A ; B)$. Unfortunately, the mutual information can only provide an upper bound on $K_{S}$ that is not really tight.

Intrinsic information $I(A ; B \downarrow E)$ : It provides an upper bound on $K_{S}$ for a particular classical key-agreement scenario. First, we describe the classical situation, and afterward, we adapt the upper bound to the QKD scenario.

In the classical case, Alice, Bob, and Eve have access to independent realizations of three random variables, $A, B$, and $E$, and which are described by the joint probability distribution $p_{A B E}$. The intrinsic information, denoted as $I(A ; B \downarrow E)$, constitutes an upper bound on the secret key rate $K_{S}$ [20]. The intrinsic information is defined as

$$
I(A ; B \downarrow E)=\inf _{E \rightarrow \bar{E}} I(A ; B \mid \bar{E}),
$$

where the minimization runs over all possible classical channels $E \rightarrow \bar{E}$ characterized by the transition probability $P_{\bar{E} \mid E}$, and where $I(A ; B \mid \bar{E})$ is the mutual information between Alice and Bob given the public announcement of Eve's data based on the probabilities $P_{A B \bar{E}}$. This quantity is defined in terms of the conditional Shannon entropy $H(X \mid \bar{e})=\sum_{x \in X}-p(x \mid \bar{e}) \log _{2} p(x \mid \bar{e})$ as

$$
I(A ; B \mid \bar{E})=\sum_{\bar{e} \in \bar{E}} P(\bar{e})[H(A \mid \bar{e})+H(B \mid \bar{e})-H(A, B \mid \bar{e})] .
$$

The intrinsic information satisfies 20]

$$
0 \leq K_{S} \leq I(A ; B \downarrow E) \leq I(A ; B) .
$$

That is, $I(A ; B \mid \bar{E})$ is a tighter bound on $K_{S}$ than the mutual information. However, recently it has been proven that the secret key rate can be smaller than the intrinsic information 21, 22.

More important for QKD, the upper bound based on the intrinsic information can be adapted to the case where Alice, Bob, and Eve start sharing a tripartite quantum state instead of a joint probability distribution. For this purpose, one can consider all possible tripartite states that Eve can establish using her eavesdropping method, and all possible measurements she could perform on her subsystem. This gives rise to a set of possible extensions $\mathcal{P}$ of the probability distribution $P_{A B}$ to $P_{A B E}$. Now one can define the intrinsic information as [7]

$$
I(A ; B \downarrow E)=\inf _{\mathcal{P}} I(A ; B \mid E) .
$$

As in the case of the mutual information, also this bound allows us to include the imperfections of the detection devices when calculating $I(A ; B \downarrow E)$. Moreover, it provides effective entanglement verification, i.e., $I(A ; B \downarrow E)=0$ if and only if the equivalence class $\mathcal{S}$ contains a separable state [7, 16]. Unfortunately, it requires us to solve an infimum problem that is not easy to compute. Note that if the range of the random variable $E$ is finite, then the infimum becomes a minimum over channels with the same alphabet [17].

Regularized relative entropy of entanglement $E_{r}^{\infty}\left(\rho_{A B}\right): \quad$ Suppose Alice and Bob share several copies of a quantum state $\rho_{A B}$, and they are allowed to perform arbitrary local operations and classical communication (LOCC). The regularized relative entropy of entanglement $E_{r}^{\infty}\left(\rho_{A B}\right)$ is an upper bound on $K_{S}$ [1]. The relative entropy of entanglement $E_{r}\left(\rho_{A B}\right)$ is given by $[12,23$

$$
E_{r}\left(\rho_{A B}\right)=\inf _{\sigma_{s e p}} \operatorname{Tr}\left[\rho_{A B}\left(\log \rho_{A B}-\log \sigma_{s e p}\right)\right] .
$$

where the infimum is taken over all separable states $\sigma_{\text {sep }}$. The regularized version of $E_{r}\left(\rho_{A B}\right)$ is given by [14, 24]:

$$
E_{r}^{\infty}\left(\rho_{A B}\right)=\lim _{n \rightarrow \infty} \frac{E_{r}\left(\rho_{A B}^{\otimes n}\right)}{n} .
$$

This quantity depends only on the shared quantum states $\rho_{A B}$, and therefore it does not include the possible imperfections on the detectors of Alice and Bob. Moreover, to calculate the regularized relative entropy of entanglement of a given quantum state is, in general, a quite difficult task, and analytical expressions are only available for some particular states [14].

Intrinsic information of a tripartite quantum state $I\left(\rho_{A B E}\right)$ : Assume Alice, Bob, and Eve share several copies of a quantum state $\rho_{A B E}$, and they are allowed to perform arbitrary LOCC and may communicate via a public channel. The intrinsic information of the tripartite quantum state $\rho_{A B E}$ is defined as 15

$$
I\left(\rho_{A B E}\right)=\inf _{E_{k}} \sum_{k} p\left(e_{k}\right) S(A ; B)_{e_{k}},
$$

with $S(A ; B)_{e_{k}}=S\left(\rho_{A}^{e_{k}}\right)+S\left(\rho_{B}^{e_{k}}\right)-S\left(\rho_{A B}^{e_{k}}\right)$ being the quantum mutual information of the conditional state $\rho_{A B}^{e_{k}}=\operatorname{Tr}_{E}\left(E_{k} \rho_{A B E}\right) / p\left(e_{k}\right)$, and represents an upper bound on $K_{S}$ [15]. Here $S(\rho)$ denotes the von Neumann entropy of the state $\rho$. It is defined as $S(\rho) \equiv$ $-\operatorname{Tr}\left(\rho \log _{2} \rho\right)$. The infimum given in Eq. (9) runs over all possible POVMs $\left\{E_{k}\right\}$.

This upper bound depends only on the quantum state $\rho_{A B E}$ and its definition does not include the effect of possible imperfections in the detectors of Alice and Bob in the most general case.

\section{UPPER BOUND ON $K_{S}$}

In this section, we introduce a simple procedure, cf. Sec.IIID to obtain an upper bound on $K_{S}$ that satisfies 
the two desired conditions presented in Sec. II. Moreover, this procedure has the advantage that it is straightforward to calculate. In order to do that, we start by presenting a naive method which allows the derivation of a simple upper bound on $K_{S}$. This method is based on imposing a particular eavesdropping strategy by Eve, and it guarantees that any resulting upper bound is able to monitor possible detector inefficiencies by construction. The necessary entanglement verification condition is then included as a particular example of this method. This is done by selecting a special eavesdropping strategy that exploits the best separable approximation (BSA) [26, 27].

\section{A. Simple method to derive upper bounds on $K_{S}$}

The idea is simple: just impose some particular eavesdropping strategy for Eve, and then use one of the already known upper bounds. The upper bound obtained represents an upper bound for any possible eavesdropping strategy. If we use as starting point, for instance, the intrinsic information, then the bound would be able to include the effect of imperfect detectors. The method can be described with the following three steps.

(1) Select a particular eavesdropping strategy for Eve. This strategy is given by the choice of a tripartite quantum state $\rho_{A B E}$ and a POVM $\left\{E_{k}\right\}$. The only restriction here is $\operatorname{Tr}_{E}\left(\rho_{A B E}\right) \in \mathcal{S}$. That is, the chosen strategy cannot alter the observed distribution $p_{i j}$.

(2) Select three random variables $A, B$, and $E$ with probability distribution $p_{i j k}=\operatorname{Tr}\left(A_{i} B_{j} E_{k} \rho_{A B E}\right)$. Note that the POVMs of Alice $\left\{A_{i}\right\}$ and Bob $\left\{B_{j}\right\}$ are known and cannot be modified by Eve.

(3) Calculate the intrinsic information $I_{E_{k}}(A ; B \downarrow E)$, where the subscript $E_{k}$ denotes the chosen measurement strategy for Eve. The secret key rate $K_{S}$ is upper bounded by $I_{E_{k}}(A ; B \downarrow E)$.

Step (3) could also be substituted by an optimization taken over all possible measurement strategies performed by Eve. That is, one might use as upper bound [16]

$$
K_{S} \leq \inf _{E_{k}} I_{E_{k}}(A ; B \downarrow E)=\inf _{E_{k}} I_{E_{k}}(A ; B \mid E) .
$$

Moreover, in this last case, it is easy to see that the same upper bound could also be obtained from the intrinsic information of a tripartite quantum state. The corresponding steps for this case are included in App. A

This method does not always guarantee effective entanglement verification, but this depends on the eavesdropping strategy selected in step (1). In Sec. IIIC we introduce a special eavesdropping strategy that guarantees that this property is always satisfied. It is based on the use of the BSA of a quantum state [26, 27]. Next, we introduce the equivalence between the BSA idea and effective entanglement verification.

\section{B. Best separable approximation and necessary entanglement verification}

The best separable approximation (BSA) [26, 27] of a given state $\rho_{A B}$ is the decomposition of $\rho_{A B}$ into a separable state $\sigma_{\text {sep }}$ and an entangled state $\rho_{\text {ent }}$, while maximizing the weight of the separable part. That is, given an arbitrary state $\rho_{A B}$, it can be proven that this state can always be written in a unique way as

$$
\rho_{A B}=\lambda_{\max }\left(\rho_{A B}\right) \sigma_{s e p}+\left[1-\lambda_{\max }\left(\rho_{A B}\right)\right] \rho_{e n t},
$$

where the entanglement state $\rho_{\text {ent }}$ has no product vectors in its range, and the real parameter $\lambda_{\max }\left(\rho_{A B}\right) \geq 0$ is maximal [26, 27].

Given an equivalence class $\mathcal{S}$ of quantum states, one defines the maximum weight of separability within the equivalence class, $\lambda_{\max }^{\mathcal{S}}$, as

$$
\lambda_{\max }^{\mathcal{S}}=\max \left\{\lambda_{\max }\left(\rho_{A B}\right) \mid \rho_{A B} \in \mathcal{S}\right\} .
$$

This parameter is related to the task of effective entanglement verification by the following observation.

Observation 1. Assume that Alice and Bob can perform local measurements with POVM elements $A_{i}$ and $B_{j}$, respectively, to obtain the probability distribution of the outcomes $p_{i j}$ on the distributed quantum state $\rho_{A B}$. Then the following two statements are equivalent: (1) The correlations $p_{i j}$ can originate from a separable state. (2) The maximum weight of separability $\lambda_{\max }^{\mathcal{S}}$ within the equivalence class of quantum states $\mathcal{S}$ compatible with the observed data $p_{i j}$ satisfies $\lambda_{\max }^{\mathcal{S}}=1$.

Proof. If $p_{i j}$ can originate from a separable state, then there exists $\sigma_{\text {sep }}$ such as $\sigma_{\text {sep }} \in \mathcal{S}$. Moreover, we have that any separable state satisfy $\lambda_{\max }\left(\sigma_{\text {sep }}\right)=1$. The other direction is trivial.

Let us define $\mathcal{S}_{\max }$ as the equivalence class of quantum states composed by those states $\rho_{A B} \in \mathcal{S}$ with maximum weight of separability

$$
\mathcal{S}_{\max }=\left\{\rho_{A B} \in \mathcal{S} \mid \lambda_{\max }\left(\rho_{A B}\right)=\lambda_{\max }^{\mathcal{S}}\right\} .
$$

\section{Eavesdropping strategy}

Eve's eavesdropping strategy is completely characterized by selecting a tripartite quantum state $\rho_{A B E}$ and a POVM $\left\{E_{k}\right\}$. We consider a pure state $\rho_{A B E}=$ $|\Phi\rangle_{A B E}\langle\Phi|$ that is a purification of a state $\rho_{A B}$ chosen from the equivalence class $\mathcal{S}_{\max }$.

We can write the separable part $\sigma_{\text {sep }}$ and the entangled part $\rho_{\text {ent }}$ of the BSA of $\rho_{A B}$ as

$$
\begin{aligned}
\sigma_{\text {sep }} & =\sum_{i} q_{i}\left|\phi_{i}\right\rangle_{A}\left\langle\phi_{i}|\otimes| \varphi_{i}\right\rangle_{B}\left\langle\varphi_{i}\right|, \\
\rho_{\text {ent }} & =\sum_{i} p_{i}\left|\psi_{i}\right\rangle_{A B}\left\langle\psi_{i}\right| .
\end{aligned}
$$


The tripartite state $|\Phi\rangle_{A B E}$ is then given by

$$
\begin{aligned}
|\Phi\rangle_{A B E}= & \sum_{i} \sqrt{\lambda_{\text {max }}^{\mathcal{S}} q_{i}}\left|\phi_{i}\right\rangle_{A}\left|\varphi_{i}\right\rangle_{B}\left|e_{i}\right\rangle_{E}+ \\
& \sum_{j} \sqrt{\left(1-\lambda_{\max }^{\mathcal{S}}\right) p_{j}}\left|\psi_{j}\right\rangle_{A B}\left|f_{j}\right\rangle_{E},
\end{aligned}
$$

where the states $\left\{\left|e_{i}\right\rangle_{E},\left|f_{j}\right\rangle_{E}\right\}$ form an orthogonal basis on Eve's subsystem. It is important to note that in both kinds of QKD schemes, EB schemes and PM schemes, Eve can have access to the state $|\Phi\rangle_{A B E}$ given by Eq. [16 []. In an EB scheme, this is clear since Eve is the one who prepares the state $\rho_{A B}$ and who distributes it to Alice and Bob. In the case of PM schemes, we need to show additionally that the state $|\Phi\rangle_{A B E}$ can be obtained by Eve by interaction with Bob's system only. In the Schmidt decomposition, the state prepared by Alice, $\left|\psi_{\text {source }}\right\rangle_{A B}$, can be written as $\left|\psi_{\text {source }}\right\rangle_{A B}=$ $\sum_{i} c_{i}\left|u_{i}\right\rangle_{A}\left|v_{i}\right\rangle_{B}$. Then the Schmidt decomposition of $|\Phi\rangle_{A B E}$, with respect to system $A$ and the composite system $B E$, is of the form $|\Phi\rangle_{A B E}=\sum_{i} c_{i}\left|u_{i}\right\rangle_{A}\left|\tilde{e}_{i}\right\rangle_{B E}$, since $c_{i}$ and $\left|u_{i}\right\rangle_{A}$ are fixed by the known reduced density matrix $\rho_{A}$ to the corresponding values of $\left|\psi_{\text {source }}\right\rangle_{A B}$. Then one can find a suitable unitary operator $U_{B E}$ such that $\left|\tilde{e}_{i}\right\rangle_{B E}=U_{B E}\left|v_{i}\right\rangle_{B}|0\rangle_{E}$ where $|0\rangle_{E}$ is an initial state of an auxiliary system.

As a measurement strategy for Eve, we consider that she is restricted to use a special class of measurements. This class of measurements can be thought of as a two step procedure.

(1) First, Eve distinguishes contributions coming from the separable part and from the entangled part of $\rho_{A B}$ : $\sigma_{\text {sep }}$ and $\rho_{\text {ent }}$, respectively. This corresponds to a projection of Eve's subsystem onto the orthogonal subspaces $\Pi_{s e p}=\sum_{i}\left|e_{i}\right\rangle_{E}\left\langle e_{i}\right|$ and $\Pi_{e n t}=\sum_{j}\left|f_{j}\right\rangle_{E}\left\langle f_{j}\right|$.

(2) Afterward, she performs a refined measurement on each subspace separately. In the separable subspace, Eve can obtain complete information, and no secret key can be distilled by Alice and Bob [7, 16]. This corresponds to a projection onto the orthogonal quantum states $\left\{\left|e_{i}\right\rangle\right\}$. In the entanglement part, Eve performs a POVM denoted as $\left\{F_{l}\right\}$.

We find that Eve's measurement result belongs to the separable subspace with probability $\lambda_{\max }^{\mathcal{S}}$, and therefore, $K_{S}=0$.

With probability $1-\lambda_{\max }^{\mathcal{S}}$, Eve's subsystem is in the entanglement subspace and $K_{S}$ might be bigger than zero. After the first step of Eve's measurement, the conditional quantum state of Alice, Bob, and Eve, denoted as $\rho_{A B E}^{e n t}=\left|\Phi_{e n t}\right\rangle_{A B E}\left\langle\Phi_{e n t}\right|$, corresponds to a purification of $\rho_{\text {ent }}$, i.e.,

$$
\left|\Phi_{\text {ent }}\right\rangle_{A B E}=\sum_{j} \sqrt{p_{j}}\left|\psi_{j}\right\rangle_{A B}\left|f_{j}\right\rangle_{E} .
$$

Note that both steps can be described as well together by the following POVM: $\left\{\tilde{E}_{\text {sep }}^{i}, \tilde{E}_{\text {ent }}^{l}\right\}$, with $\tilde{E}_{\text {sep }}^{i}=$ $\left|e_{i}\right\rangle\left\langle e_{i}\right|$ and $\tilde{E}_{\text {ent }}^{l}=F_{l} \Pi_{\text {ent }}$.
In the next section, we provide an upper bound for $K_{S}$ that arises from this special eavesdropping strategy. Moreover, as we will see, the upper bound obtained is straightforward to calculate.

\section{Resulting upper bound}

Only the entangled part $\rho_{\text {ent }}$ which appears in the BSA decomposition of a given state $\rho_{A B}$ might allow Alice and Bob to distill a secret key in the eavesdropping strategy proposed in the previous section. Moreover, Eve can always find such an eavesdropping strategy for any $\rho_{A B} \in \mathcal{S}_{\max }$. This fact motivates the definition of a new equivalence class of quantum states $\mathcal{S}_{\max }^{\text {ent }}$

$$
\left.\mathcal{S}_{\text {max }}^{\text {mat }}=\left\{\rho_{\text {ent }}\left(\rho_{A B}\right) \mid \rho_{A B} \in \mathcal{S}_{\max }\right)\right\}
$$

where $\rho_{\text {ent }}\left(\rho_{A B}\right)$ denotes the entangled part in the BSA of the state $\rho_{A B}$.

Theorem 1. Consider all possible bipartite entanglement states $\rho_{\text {ent }} \in \mathcal{S}_{\text {max }}^{\text {ent }}$, and consider all possible POVMs $\left\{F_{l}\right\}$ that Eve could perform on a purification $\left|\Phi_{\text {ent }}\right\rangle_{A B E}$ of the quantum state $\rho_{\text {ent }}$. This gives rise to a set $\mathcal{P}$ of tripartite probability distributions $p_{i j l}=$ $\operatorname{Tr}\left(A_{i} B_{j} F_{l}\left|\Phi_{\text {ent }}\right\rangle_{A B E}\left\langle\Phi_{\text {ent }}\right|\right)$, where $\left\{A_{i}\right\}$ and $\left\{B_{j}\right\}$ represent the POVMs measured by Alice and Bob. The secret key rate $K_{S}$ is upper bounded by

$$
K_{S} \leq\left(1-\lambda_{\max }^{\mathcal{S}}\right) \inf _{\mathcal{P}} I^{e n t}(A ; B \mid E)
$$

where $I^{\text {ent }}(A ; B \mid E)$ represents the classical conditional mutual information of three random variables distributed accordingly to $p_{i j l}$.

Proof: This proof is straightforward since, by construction, Eq. (19) is an upper bound of the intrinsic information defined in Eq. (6). Note that to obtain Eq. (19), we assume a particular type of eavesdropping for Eve, and in Eq. (6), the infimum is taken over all possible eavesdropping strategies.

Instead of optimizing over all possible states $\rho_{A B} \in$ $\mathcal{S}_{\text {max }}^{\text {ent }}$ and all possible measurements performed by Eve, one could also take any state in $\mathcal{S}_{\text {max }}^{\text {ent }}$ and calculate the infimum of $I^{\text {ent }}(A ; B \mid E)$ over all possible POVMs $\left\{F_{l}\right\}$ employed by Eve. This fact simplifies the calculation of the upper bound on $K_{S}$.

Corollary 1. Given a state $\rho_{\text {ent }} \in \mathcal{S}_{\text {max }}^{\text {ent }}$, the secret key rate $K_{S}$ is upper bounded by

$$
K_{S} \leq\left(1-\lambda_{\max }^{\mathcal{S}}\right) \inf _{F_{l}} I_{F_{l}}^{e n t}(A ; B \mid E),
$$

with $I_{F_{l}}^{e n t}(A ; B \mid E)$ being the classical conditional mutual information calculated on the probability distribution $p_{i j l}=\operatorname{Tr}\left(A_{i} B_{j} F_{l}\left|\Phi_{\text {ent }}\right\rangle_{A B E}\left\langle\Phi_{\text {ent }}\right|\right)$, and where $\left|\Phi_{\text {ent }}\right\rangle_{A B E}$ denotes a purification of $\rho_{\text {ent }}$.

Proof. Equation (20) is an upper bound of Eq. (19). Note that in Eq. (20), we take a particular state $\rho_{\text {ent }} \in$ $\mathcal{S}_{\text {max }}^{\text {ent }}$, while the infimum in Eq. (19) includes all possible states $\rho_{\text {ent }} \in \mathcal{S}_{\text {max }}^{\text {ent }}$. 
The upper bounds provided by Theorem 1 and Corollary 1 are easier to calculate than the one based on the intrinsic information defined in Eq. (6) 28]. However, they still demand solving a difficult optimization problem. Next, we provide a simple upper bound on $K_{S}$ that is straightforward to calculate. Then, in Sec. IV we illustrate the performance of this upper bound for two well-known QKD protocols with imperfect detectors: the four-state [2] and the six-state [18] QKD schemes. We compare it with the upper bound given by the regularized relative entropy of entanglement 11]. by

Corollary 2: The secret key rate $K_{S}$ is upper bounded

$$
K_{S} \leq\left(1-\lambda_{\max }^{\mathcal{S}}\right) I^{e n t}(A ; B),
$$

where $I^{\text {ent }}(A ; B)$ denotes the mutual information calculated on the probability distribution $\tilde{p}_{i j}=\operatorname{Tr}\left(A_{i} B_{j} \rho_{\text {ent }}\right)$ with $\rho_{\text {ent }} \in \mathcal{S}_{\text {max }}^{\text {ent }}$.

Proof: Equation (21) is an upper bound of Eq. (20), i.e., $\inf _{F_{l}} I_{F_{l}}^{\text {ent }}(A ; B \mid E) \leq I^{\text {ent }}(A ; B)$. Note that Eve could always select a POVM with only one element $\tilde{F}_{l}=\mathbb{1}$. In this case, we have $I_{\tilde{F}_{l}}^{\text {ent }}(A ; B \mid E)=I^{\text {ent }}(A ; B)$.

In Sec. IV we show that, for qubit-based QKD protocols, the upper bound given by Corollary 1 and by Corollary 2 coincide.

The main difficulty when evaluating this last upper bound for a particular realization of QKD relies on obtaining $\lambda_{\max }^{\mathcal{S}}$ and $\rho_{\text {ent }}$. This can be solved by applying results from relaxation theory of nonconvex problems [29, 30, 31. See also Ref. 32]. The solution is included in App. B

The three bounds on $K_{S}$ obtained in this section satisfy the two desired properties included in Sec. IA the factor $1-\lambda_{\max }^{\mathcal{S}}$ accounts for effective entanglement verification by Observation 1, and the probabilities $p_{i j l}$ and $\tilde{p}_{i j}$ reflect possible detector inefficiencies.

\section{EVALUATION OF THE UPPER BOUND}

In this section, we evaluate the upper bound on $K_{S}$ given by Eq. (21) for two well-known qubit-based QKD protocols with imperfect detectors: the four-state 2] and the six-state [18] QKD schemes. We refer here to singlephoton implementations of the qubit. The state of the qubit is described by some degree of freedom in the polarization of the photon.

We start by describing the detection devices employed by Alice and Bob. They are characterized by some noise in the form of dark counts which are, to a good approximation, independent of the incoming signals, and by their detection efficiency $\eta$.

Dark counts: For simplicity, we assume that only Bob's detection device is affected by the presence of dark counts. We consider that Alice's detectors are ideal. The total dark count probability is denoted as $d$. A typical value for this parameter is $d=10^{-6}$. This scenario can be modeled by transforming every ideal POVM element $B_{j}$ of Bob into a noisy element $\tilde{B}_{j}$ given by

$$
\tilde{B}_{j}=(1-d) B_{j}+d_{j} \mathbb{1}_{B},
$$

where the parameters $d_{j}$ satisfy $d=\sum_{j} d_{j}$.

The effect of the noise can be included in the calculation of the upper bound given by Eq. (21) via the classical mutual information $I^{e n t}(A ; B)$. We have that $I^{e n t}(A ; B)>I^{e n t}(A ; \tilde{B})$ simply because the raw data is affected by more noise which needs to be corrected.

It is important to note that the effective entanglement verification alone cannot include the noise coming from the dark counts of the detectors. In the ideal case, Alice and Bob observe outcomes governed by the ideal probability distribution $p_{i j}$, which defines the equivalence class of quantum states $\mathcal{S}$. In the noisy scenario, on the contrary, Alice and Bob obtain the probability distribution $\tilde{p}_{i j}=(1-d) p_{i j}+d_{j} p_{i}$ defining an equivalence class $\tilde{\mathcal{S}}$. In principle, both probability distributions are different. However, in the trusted device scenario, Alice and Bob know all the parameters $d_{j}$. This means that they can obtain $p_{i j}$ from $\tilde{p}_{i j}: p_{i j}=1 /(1-d)\left(\tilde{p}_{i j}-d_{j} p_{i}\right)$, and where $p_{i}=\sum_{j} \tilde{p}_{i j}[33$. That is, we obtain $\tilde{\mathcal{S}}=\mathcal{S}$ : any upper bound which only depends on the shared quantum state $\rho_{A B}$ provides the same upper bound independently of the noise introduced by the detectors.

Detector efficiency: Detectors are characterized as well by their detector efficiency $\eta_{j}$. This effect can be modeled by a combination of a beam splitter of transmittance $\eta_{j}$ and an ideal detector [34]. A typical value for $\eta_{j}$ in current realizations of QKD is approximately 0.15 . In order to include the losses of the detectors, we can transform every POVM element $B_{j}$ that corresponds to a "click" event into

$$
\tilde{B}_{j}=\eta_{j} B_{j}
$$

Additionally, the event "no click" corresponds to the following operator:

$$
B_{v a c}=\sum_{j}\left(1-\eta_{j}\right) B_{j}+|\mathrm{vac}\rangle\langle\mathrm{vac}| .
$$

where $\mid$ vac $\rangle$ represents the vacuum state.

As in the case of dark counts, the effect of the losses is incorporated in Eq. (21) via the classical mutual information $I^{e n t}(A ; B)$. For simplicity, we consider that $\eta_{j}=\eta$ for all the detectors.

In Fig. 11 we illustrated the upper bound given by Eq. (21) for the four-state [2] and the six-state [18] QKD schemes. In the case of the four-state EB protocol, Alice and Bob perform projection measurements onto two mutually unbiased bases, say the ones given by the eigenvectors of the two Pauli operators $\sigma_{x}$ and $\sigma_{z}$. In the corresponding PM scheme, Alice can use as well the same set of measurements but now on a maximally entangled state. For the case of the six-state EB protocol, Alice and 


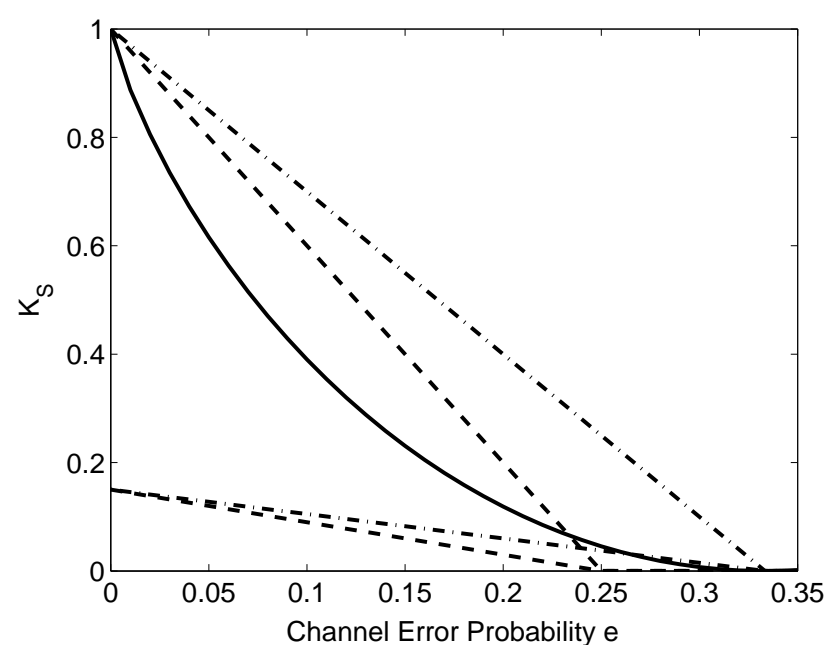

FIG. 1: Upper bound on the secret key rate $K_{S}$ given by Eq. (21) for the four-state (dashed) and the six-state (dash-dotted) QKD schemes. The classical correlated data $p_{i j}$ is obtained by measuring the quantum state $\rho_{A B}(e)=$ $(1-2 e)\left|\psi^{+}\right\rangle\left\langle\psi^{+}\right|+e \mathbb{1} / 2$, where $e$ is the error probability of a depolarizing channel. The two upper lines (dashed and dash-dotted) correspond to the ideal case where the total dark count probability is $d=0$, and the detector efficiency is $\eta=1$. The two lines below represent the typical case in current realizations of QKD: $d=10^{-6}$ and $\eta=0.15$. The solid line of the graphic represent the upper bound for $K_{S}$ given by the regularized relative entropy of entanglement $E_{r}^{\infty}\left(\rho_{A B}(e)\right)$. Note that we assume an asymmetric basis choice to suppress the sifting effect [36].

Bob perform projection measurements onto the eigenvectors of the three Pauli operators $\sigma_{x}, \sigma_{y}$, and $\sigma_{z}$ on the bipartite qubit states distributed by Eve. In the corresponding PM scheme Alice prepares the eigenvectors of those operators by performing the same measurements on a maximally entangled two-qubit state. Note that here we are not using the general approach introduced previously, $\left|\psi_{\text {source }}\right\rangle_{A B}=\sum_{i} \sqrt{p_{i}}\left|\alpha_{i}\right\rangle_{A}\left|\varphi_{i}\right\rangle_{B}$, to model PM schemes, since for this protocol it is sufficient to consider that the effectively distributed quantum state consists only of two qubits.

We model the transmission channel as a depolarizing channel with error probability $e$. This means that, in both protocols, the joint probability distribution $p_{i j}$ is obtained by measuring the quantum state

$$
\rho_{A B}(e)=(1-2 e)\left|\psi^{+}\right\rangle\left\langle\psi^{+}\right|+\frac{e}{2} \mathbb{1},
$$

where the state $\left|\psi^{+}\right\rangle$represents a maximally entangled two-qubit state: $\left|\psi^{+}\right\rangle=1 / \sqrt{2}(|00\rangle+|11\rangle)$. In Fig. 1] we assume as well that the total dark count probability is $d=10^{-6}$, and the detector efficiency is $\eta=0.15$. Moreover, we consider that all detectors have the same dark count probability.

It is important to note that in this simple twoqubit scenario, the conditional mutual information
$I_{F_{l}}^{e n t}(A ; B \mid E)$ reduces to $I^{e n t}(A ; B)$ : in this case $\rho_{\text {ent }}$ is just a pure entangled state 26], i.e., the purification $\left|\Phi_{\text {ent }}\right\rangle$ of the quantum state $\rho_{\text {ent }}$ is of the form $\left|\Phi_{\text {ent }}\right\rangle=$ $|\psi\rangle_{A B} \otimes|\varphi\rangle_{E}$. For qubit-based QKD protocols, therefore, the upper bound given by Corollary 1 and by Corollary 2 coincide since $\inf _{F_{l}} I_{F_{l}}^{e n t}(A ; B \mid E)=I^{e n t}(A ; B)$. In order to calculate the maximum weight of separability $\lambda_{\max }^{\mathcal{S}}$, and its associated entangled state $\rho_{\text {ent }}$ which are necessary to evaluate Eq. (21) we use the method described in Appendix B

Figure 1 includes also a comparison with the upper bound on $K_{S}$ given by the regularized relative entropy of entanglement [11]. To calculate $E_{r}^{\infty}\left(\rho_{A B}(e)\right)$, we use the results included in Ref. [14]. Despite its simplicity, the upper bound given by Eq. (21) can provide a tighter bound on $K_{S}$ than the one proposed in Ref. [11], when dealing with typical parameter values for imperfect detectors. It must be mentioned here, however, that the bound given by $E_{r}^{\infty}\left(\rho_{A B}(e)\right)$ was proposed in a different scenario, and the possibility of having noisy and lossy detectors was not considered. The results obtained can also be compared with the best lower bounds for the tolerable error rate $e$ arising from known security proofs [35]: $e=0.2$ for the case of the four-state protocol, and $e=0.276$ for the six-state protocol.

\section{CONCLUSION}

A necessary precondition for secure quantum key distribution (QKD) is that sender and receiver can use their available measurement results to prove the presence of entanglement in a quantum state that is effectively distributed between them. Moreover, this result applies both to prepare and measure and entanglement-based schemes.

Unfortunately, this effective entanglement verification answers only partially the important question of how much secret key can be obtained by Alice and Bob from their correlated data: it just tells if the secret key rate is zero or it may be positive.

In this paper, we present a simple method to obtain an upper bound on the secret key rate that is particularly suited to evaluate practical realizations of QKD schemes. It is based on the correlated data, but it also monitors any form of detector imperfections. In particular, we consider the so-called trusted device scenario, where Eve cannot modify the actual detection devices employed by Alice and Bob. We assume that the legitimate users have complete knowledge about their detectors, which are fixed by the actual experiment.

\section{ACKNOWLEDGMENTS}

The authors wish to thank M. Horodecki, M. Christandl, W. Mauerer, J. Rigas, G. O. Myhr, J. M. Renes, and K. Tamaki for very useful discussions. This work was 
supported by the DFG under the Emmy Noether programme, the European Commission (Integrated Project SECOQC).

\section{APPENDIX A: EQUIVALENT METHOD}

In this Appendix, we present an alternative method to the one introduced in Sec. IIIA that provides exactly the same upper bound on $K_{S}$. It uses the intrinsic information of a tripartite quantum state, and it can be described with the following steps:

(1) Select a particular eavesdropping strategy for Eve. This strategy is given by the choice of a tripartite quantum state $\rho_{A B E}$. The restriction here is $\operatorname{Tr}_{E}\left(\rho_{A B E}\right) \in \mathcal{S}$.

(2) Compute the so-called $c c q$ state. This state is of the form [25]:

$$
\rho_{A B E}^{c c q}=\sum_{i j}|i j\rangle_{A B}\langle i j| \otimes \rho_{E}^{i j}
$$

where the state $\rho_{E}^{i j}$ is not normalized and it is given by $\rho_{E}^{i j}=\operatorname{Tr}_{A B}\left(A_{i} B_{j} \rho_{A B E}\right)$.

(3) The upper bound on $K_{S}$ is given by the intrinsic information of the tripartite $c c q$ state $\rho_{A B E}^{c c q}$.

The equivalence of this method and the one introduced in Sec. IIA can be seen as follows: if the chosen tripartite state $\rho_{A B E}$ is the same for both methods and one fixes a measurement strategy $\left\{E_{k}\right\}$ for Eve, then the conditional quantum mutual information $S(A ; B)_{e_{k}}$ is equal to $I_{E_{k}}\left(A ; B \mid E=e_{k}\right)$. Note that in this case, the conditional state becomes $\rho_{A B}^{c c q, e_{k}}=\sum_{i j} p\left(a_{i}, b_{j} \mid e_{k}\right)|i j\rangle_{A B}\langle i j|$.

\section{APPENDIX B: OBTAINING $\lambda_{\max }^{\mathcal{S}}$ AND $\rho_{\text {ent }}$}

Here we use the method proposed in Ref. 32] to obtain the parameter $\lambda_{\max }^{\mathcal{S}}$ and the density operator $\rho_{\text {ent }}$. It is based on results from relaxation theory of nonconvex problems [29, 30, 31], notably the method of Lasserre 30. The central observation in Ref. 32 is that many problems related with entanglement can be cast into the form of optimization problems with polynomial constraints of low degree (three or less). The polynomial part of the optimization problems is still nonconvex and computationally expensive to solve. However, one can find hierarchies of solutions in a way that each step is a better approximation to the exact solution than the previous one. Moreover, each step can be efficiently solved via semidefinite programming [37]. The hierarchy is asymptotically complete, in the sense that the exact solution is asympotically attained.

It is important to note that the method introduced in Ref. [32] is not only meant as a numerical method, but each instance of the hierarchy delivers a semidefinite program that is accessible with analytical methods.
Next, we explain how to cast the problem of finding $\lambda_{\max }^{\mathcal{S}}$ and $\rho_{\text {ent }}$ into the desired form analyzed in Ref. [32].

The equivalence class of quantum states $\mathcal{S}$ is defined by the POVMs $\left\{A_{i} \otimes B_{j}\right\}$ and the observed data $p_{i j}: \rho_{A B} \geq$ 0 belongs to $\mathcal{S}$ if it satisfies $\operatorname{Tr}\left(A_{i} \otimes B_{j} \rho_{A B}\right)=p_{i j}$ for all $i, j$. The BSA for $\rho_{A B}$ can be written in the following way

$$
\rho_{A B}=\min _{\operatorname{Tr}\left(\tilde{\rho}_{\text {ent }}\right)} \tilde{\sigma}_{s e p}+\tilde{\rho}_{\text {ent }}
$$

with $\tilde{\sigma}_{\text {sep }} \equiv \lambda_{\max }\left(\rho_{A B}\right) \sigma_{\text {sep }}$ and $\tilde{\rho}_{\text {ent }} \equiv[1-$ $\left.\lambda_{\max }\left(\rho_{A B}\right)\right] \rho_{\text {ent }}$. Separable states $\tilde{\sigma}_{\text {sep }}$ can be characterized in terms of product vectors $\tilde{\sigma}_{s e p}=\sum_{i} P_{i}$, with $P_{i}=p_{i}\left|\psi_{i}\right\rangle\left\langle\psi_{i}|\otimes| \phi_{i}\right\rangle\left\langle\phi_{i}\right|$. To guarantee that the operators $P_{i}$ have the desired product form, they must satisfy the following constraints [32]:

$$
\begin{aligned}
\operatorname{Tr}\left[\operatorname{Tr}_{I}\left(P_{i}\right)^{2}\right] & =\left[\operatorname{Tr}\left(P_{i}\right)\right]^{2} \\
P_{i} & \geq 0
\end{aligned}
$$

with $I=\{A, B\}$. The associated optimisation problem can now be written as

$$
\begin{aligned}
\operatorname{minimize} & t, \\
\text { subject to } & t \geq 1-\operatorname{Tr}\left(\tilde{\sigma}_{\text {sep }}\right), \\
& \rho_{A B} \geq 0 \\
& \operatorname{Tr}\left(\rho_{A B}\right)=1, \\
& \operatorname{Tr}\left(A_{i} \otimes B_{j} \rho_{A B}\right)=p_{i j} \forall i, j, \\
& \tilde{\sigma}_{\text {sep }}=\sum_{i} P_{i}, \\
& \operatorname{Tr}\left[\operatorname{Tr}_{I}\left(P_{i}\right)^{2}\right]=\left[\operatorname{Tr}\left(P_{i}\right)\right]^{2} \forall I=A, B, \\
& P_{i} \geq 0, \\
& \rho_{A B}-\tilde{\sigma}_{\text {sep }} \geq 0,
\end{aligned}
$$

where the parameter $t$ represents the trace of the quantum state $\tilde{\rho}_{\text {ent }}$. This polynomial optimization problem can be solved with the help of Lasserre's method 30]. For these calculations, the package GloptiPoly [38] based on SeDuMi [39] is freely available. The package GloptiPoly has a number of desirable features, in particular, it provides a certificate for global optimality.

Note that in low dimensional cases $(2 \otimes 2,2 \otimes 3)$ the characterization of separable states can be simplified to those states $\tilde{\sigma}_{\text {sep }} \geq 0$ such as $\tilde{\sigma}_{\text {sep }}^{T_{B}} \geq 0$ [40], where $T_{P}$ is the partial transposition, that is, the transposition with respect to one subsystem. The problem given by Eq. (B4) can be reduced then to one containing only linear and semidefinite constraints. Problems of this form can be solved very efficiently with standard semidefinite programming modules [37]. 
[1] S. Wiesner, Sigact News 15, 78 (1983).

[2] C. H. Bennett and G. Brassard, Proceedings of the IEEE International Conference on Computers, Systems and Signal Processing, Bangalore, India (IEEE, New York, 1984), p. 175.

[3] G. S. Vernam, J. Am. Inst. Electr. Eng. 45, 109 (1926).

[4] N. Lütkenhaus, Applied Phys. B 69, 395 (1999).

[5] C. H. Bennett, G. Brassard, and N. D. Mermin, Phys. Rev. Lett. 68, 557 (1992).

[6] M. Curty, O. Gühne, M. Lewenstein, and N. Lütkenhaus, Phys. Rev. A 71, 022306 (2005).

[7] M. Curty, M. Lewenstein, and N. Lütkenhaus, Phys. Rev. Lett. 92, 217903 (2004).

[8] N. Gisin and S. Wolf, Proceedings of CRYPTO 2000, Lecture Notes in Computer Science Vol. 1800 (Springer, New York, 2000), p. 482

[9] The equivalence class $\mathcal{S}$ reduces to the trivial one, i.e., it contains only one element up to a global phase, when the measurements realized by Alice and Bob provide complete tomographic information about $\rho_{A B}$. This is the case, for instance, of the six-state QKD protocol [18]. Otherwise, $\mathcal{S}$ contains always more than one quantum state.

[10] M. Horodecki, P. Horodecki, and R. Horodecki, in Quantum information: An Introduction to Basic Theoretical Concepts and Experiments, edited by G. Alber et al. (Springer, Heidelberg, 2001), p. 151; K. Eckert, O. Gühne, F. Hulpke, P. Hyllus, J. Korbicz, J. Mompart, D. Bruß, M. Lewenstein, and A. Sanpera, in Quantum Information Processing, edited by G. Leuchs and T. Beth, (Wiley-VCH, Verlag, 2003), p. 79. See also 2nd ed. (2005).

[11] K. Horodecki, M. Horodecki, P. Horodecki, and J. Oppenheim, Phys. Rev. Lett. 94, 160502 (2005).

[12] V. Vedral and M. B. Plenio, Phys. Rev. A 57, 1619 (1998).

[13] C. H. Bennett, D. P. DiVincenzo, J. A. Smolin, and W. K. Wootters, Phys. Rev. A 54, 3824 (1996).

[14] K. Audenaert, J. Eisert, E. Jané, M. B. Plenio, S. Virmani, and B. De Moor, Phys. Rev. Lett 87, 217902 (2001).

[15] M. Christandl, and R. Renner, Proceedings of 2004 IEEE International Symposium on Information Theory (IEEE, New York, 2004), p. 135.

[16] A. Acín, and N. Gisin, Phys. Rev. Lett. 94, 020501 (2005).

[17] M. Christandl, R. Renner, and S. Wolf, Proceedings of 2003 IEEE International Symposium on Information Theory (IEEE, New York, 2003), p. 258.

[18] D. Bruß, Phys. Rev. Lett. 81, 3018 (1998).

[19] U. M. Maurer, IEEE Trans. Inf. Theory 39, 733 (1993).

[20] U. Maurer, and S. Wolf, IEEE Trans. Inf. Theory 45, 499 (1999).
[21] R. Renner, and S. Wolf, Adv. in Crypt. EUROCRYPT'03, edited by E. Biham, Lecture Notes in Computer Science Vol. 2656 (Springer-Verlag, Berlin, 2003), p. 562 .

[22] There is the conjecture that the intrinsic information represents the exact secret key rate in a slightly different scenario than the usual secret key agreement scenario. This is the so-called public Eve scenario [15]. Here Eve must choose a function that is then used to obtain $\bar{E}$ from $E$. The value of $\bar{E}$ together with the description of the function is then broadcasted to Alice and Bob.

[23] V. Vedral, M. B. Plenio, M. A. Rippin, and P. L. Knight, Phys. Rev. Lett. 78, 2275 (1997).

[24] M. Donald, M. Horodecki, and O. Rudolph, J. Math. Phys. 43, 4252 (2002).

[25] I. Devetak, and A. Winter, Proc. R. Soc. London, Ser. A 461, 207 (2005).

[26] M. Lewenstein, and A. Sanpera, Phys. Rev. Lett. 80, 2261 (1997).

[27] S. Karnas, and M. Lewenstein, J. Phys. A 34, 6919 (2001).

[28] Note that the rank of the quantum states involved in the calculation decrease, i.e., $\operatorname{rank}\left[\rho_{\text {ent }}\left(\rho_{A B}\right)\right] \leq \operatorname{rank}\left(\rho_{A B}\right)$. Moreover, we have that $\operatorname{rank}\left[\rho_{\text {ent }}\left(\rho_{A B}\right)\right] \leq(N-1)(M-$ $1)$, where $N$ and $M$ represent the dimensions of Alice's and Bob's Hilbert space, respectively [26].

[29] N.Z. Shor, Soviet J. Circuits Syst. Sci. 25, 1 (1987).

[30] J.B. Lasserre, SIAM J. Optimization 11, 796 (2001).

[31] P.A. Parrilo, Ph.D. thesis, California Institute of Technology, Pasadena, 2000 (unpublished).

[32] J. Eisert, P. Hyllus, O. Gühne, and M. Curty, Phys. Rev. A 70, 062317 (2004).

[33] In PM schemes, all the probabilities $p_{i}$ are known since the beginning. They can be obtained directly from the reduced density matrix of Alice $\rho_{A}$ and the POVM elements $A_{i}$, which are fixed.

[34] B. Yurke, Phys. Rev. A 32, 311 (1985).

[35] H. F. Chau, Phys. Rev. A 66, 060302(R) (2002).

[36] H. K. Lo, H. F. C. Chau, and M. Ardehali, Journal of Cryptology 18, 133 (2005).

[37] L. Vandenberghe and S. Boyd, SIAM Review 38, 49 (1996); C. Helmberg, Eur. J. Oper. Res. 137, 461 (2002).

[38] D. Henrion and J.B. Lasserre, ACM Trans. Math. Softw. 29, 165 (2003); See also the web page ww.laas.fr/ henrion/ software/gloptipoly/gloptipoly.html.

[39] J. F. Sturm, Optimization Methods and Software 11, 625 (1999);. See also the documentation of the software on the web page sedumi.mcmaster.ca.

[40] A. Peres, Phys. Rev. Lett. 77, 1413 (1996); M. Horodecki, P. Horodecki, and R. Horodecki, Phys. Lett. A 223, 1 (1996). 